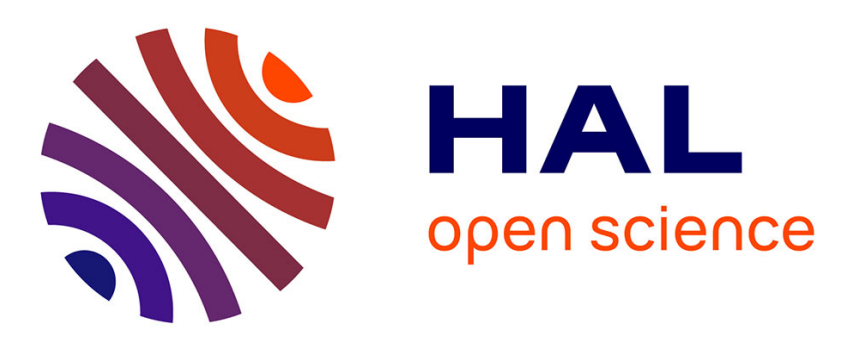

\title{
Design-oriented EMC analysis of wiring systems
}

\author{
A. Mori, P. Di Bartolomeo, M. Bandinelli, A. Bonsignore, Nathanael Muot, \\ Christophe Girard, Guillaume Prin, Jean-Phillipe Parmantier, Isabelle \\ Junqua, Solange Bertuol, et al.
}

\section{- To cite this version:}

A. Mori, P. Di Bartolomeo, M. Bandinelli, A. Bonsignore, Nathanael Muot, et al.. Design-oriented EMC analysis of wiring systems. 2020 International Symposium on Electromagnetic Compatibility EMC EUROPE, Sep 2020, Rome, Italy. pp.1-6, 10.1109/EMCEUROPE48519.2020.9245716 . hal03016821v1

\section{HAL Id: hal-03016821 \\ https://hal.science/hal-03016821v1}

Submitted on 20 Nov 2020 (v1), last revised 4 Jun 2021 (v2)

HAL is a multi-disciplinary open access archive for the deposit and dissemination of scientific research documents, whether they are published or not. The documents may come from teaching and research institutions in France or abroad, or from public or private research centers.
L'archive ouverte pluridisciplinaire HAL, est destinée au dépôt et à la diffusion de documents scientifiques de niveau recherche, publiés ou non, émanant des établissements d'enseignement et de recherche français ou étrangers, des laboratoires publics ou privés. 


\section{Design-oriented EMC analysis of wiring systems}

\author{
A. Mori, P. di Bartolomeo, M. \\ Bandinelli, A. Bonsignore \\ I.D.S. Ingegneria dei Sistemi S.p.A. \\ Pisa, Italy \\ \{a.mori,p.dibartolomeo,m.bandinelli,a. \\ bonsignore\}@idscorporation.com \\ G. Antonini, M. D. Astorino \\ Università degli Studi dell'Aquila \\ L'Aquila, Italy \\ giulio.antonini@univaq.it, \\ mariadenise.astorino@uniroma1.it
}

\author{
N. Muot, C. Girard, G. Prin \\ AxesSim \\ Illkirch-Graffenstaden, France \\ \{nathanael.muot, christophe.girard, \\ guillaume.prin\}@axessim.fr \\ C. Jullien \\ Safran Electrical \& Power \\ Blagnac, France \\ charles.jullien@safrangroup.com
}

\author{
J. P. Parmantier, I. Junqua, S. Bertuol, \\ J. Morio \\ ONERA / DEMR Université de \\ Toulouse \\ Toulouse, France \\ \{Jean- \\ Philippe.Parmantier,Isabelle.Junqua,Sol \\ ange.Bertuol,Jerome.Morio\}@onera.fr
}

\begin{abstract}
Modern aircraft hosts a large number of electric systems. Due to the transition to a More Electrical Aircraft, future platforms will use even more electric systems. This increases the complexity of the EWIS design from an EMC point of view. When possible, segregation between potentially interfering cables or bundles is a convenient solution to avoid EMC problems. Due to the huge number of installation configurations, the evaluation of the minimal segregation distance between two interacting bundles can be unfeasible. To overcome this situation, a specialized tool has been developed, based on a statistical approach for the analysis of the interaction between couples of bundles. In the paper, the different modules needed for this analysis are detailed and described. Some canonical analysis are then reported to show the capabilities of the tool.
\end{abstract}

\section{Keywords-Multiconductor-Transmission-Line-Network, Electric Wiring Interconnection System, Cable-Bundles, Statistical Method}

\section{INTRODUCTION}

Modern aircraft employs a large number of electric systems, and the current trend is to have a More Electric Aircraft. This means the operation of a very high number of potentially interacting systems with a complex Electric Wiring Interconnecting System (EWIS). This represents a challenging EMC problem, especially in the design of EWIS for which weight and cost constraints have to be considered. Different solutions can be adopted to avoid EMC problems for harnesses:

- Some cable-bundles can be shielded, at the expenses of higher cost and mass weight

- Interacting cables can be segregated in specific bundles by means an appropriate routing. If possible, segregation represents a very convenient solution, both in terms of cost and mass points of view.

However, conversely, the selection of an optimal routing - or, in other terms, the finding of the minimum distance of segregation - for the bundles constituting the EWIS is usually a challenging problem, due to the large range of variations of the installation parameters and due to the complex nature of bundle-configurations preventing the use of analytical solutions.

After the definition of these minimum distances of two routes, the problem is to obtain an acceptable 3D-design and installation of the aircraft. Between the original design of the harness and the real path in the aircraft, some modifications of the position of equipment on board make impossible the harness installation as long as the aircraft manufacturer and the certification body do not validate the derogation distance from the rule.

The aim of the ANALYST project [1] is to develop a software tool able to assess the optimal segregation distance between two cable-bundles to ensure the compatibility of the connected systems and to be able to estimate the confidence level in case of derogation from this optimal distance. The first phase of the project, reported in this paper, has resulted in the evaluation of pertinent statistical techniques. Such techniques and the operation of the developed tool are described in this paper, along with some results obtained on test-cases. In particular, Section II describes the problem to solve. Section III explains briefly the selected statistical techniques. Section IV and V describes the tool and report some results, respectively.

\section{PROBLEM STATEMENT}

The problem of finding the set of the minimum segregation distances for a set of bundles has been stated as finding the minimum distance between each couple of bundles to assure there is no unwanted interference defined by a frequency dependent susceptibility level. The level of interference between two bundles depends on the distance between the bundles, on the distances of each bundle from the nearest electrical reference (ground reference), on the coupling length and the crossing angles between them. The evaluation of the exploited methods refers to the generic geometrical configuration shown in Fig. 1. In general, we can have a couple of bundles crossing with a certain angle $\alpha$, but the most relevant configuration (more stringent in terms of cross-coupling) is the one shown at the bottom of Fig. 1, when $\alpha=0$ and the two bundles become parallel on their whole coupling length.

When the wire-sources, end-loads and the susceptibility levels are given, a specified configuration can be analyzed in the frequency domain by means of a MLTN solver based on the BLT equation [2]. The huge number of possible configurations has led to the employment of particular statistical methods to reduce the size of the problem. Strictly related to the problem of the evaluation of the minimum segregation distance, there is a second problem motivated by the request for exemption from the cable design team in case 

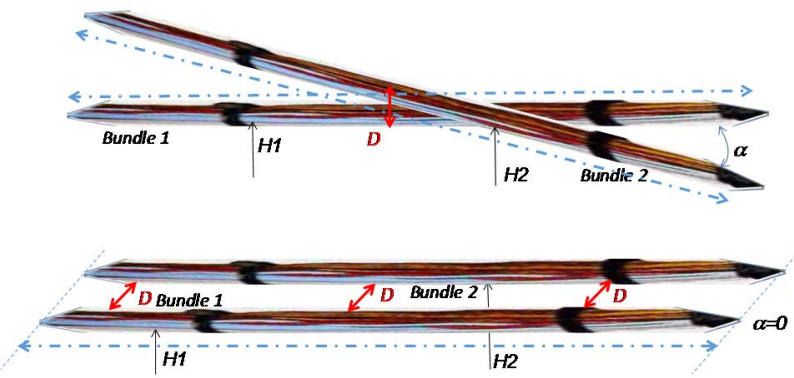

Fig. 1. Geometrical configuration of the two-bundles problems: Top: General configuration: bundle pair crossing with an $\alpha$ angle. Bottom: Pair of bundles uniformly parallel along their whole length.

it is impossible to respect the minimum distance constraint in a real aircraft installation. In such a case, the selected statistical methods can be used to estimate the confidence of compliance. The next section briefly describes the selected statistical methods.

\section{StATISTICAL ANALYSIS OF EM SUSCEPTIBILITY}

Given the generators and loads on the cables of the two bundles, the MLTN model solves the two-bundles problem in the frequency domain. The voltages and currents at the endload levels are then compared with pertinent susceptibility level to verify compliance. In particular, in order to set up an efficient optimization process, we define the $G$ scalar quantity:

$$
G\left(D, l, H_{A}, H_{B}\right)=\max _{f, n A, n B}\left[\frac{o_{A, i}-S_{A, i}}{S_{A, i}}, \frac{o_{B, j}-S_{B, j}}{S_{B, j}}\right],
$$

where $O_{A, i}, O_{B, j}$ are either voltages or currents on a wire $i$ of bundle $A$ or a wire $j$ of bundle $B$, depending from the definition of the susceptibility level $S_{A, i}, S_{B, j}$. The maximum value over the whole frequency sweep $\{f\}$ is chosen to define $G$. For a particular configuration (i.e. for a specified set of installation parameters, $D, l, H_{A}, H_{B}$ ), a value $G>0$ indicates an interfering configuration. The problem of finding the minimum segregation distance consists then in evaluating the minimum value of $D=D_{\min }$ so that

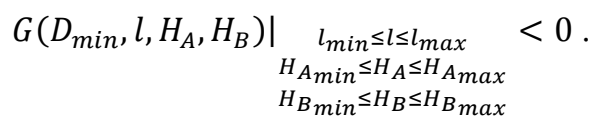

The large number of possible configurations (i.e. of combinations of the installation parameters) makes a direct computation unfeasible from an operational point of view. Hopefully, statistical methods are able to describe the behavior of the $G$ function over the wide range of possible configurations. By using a statistical approach, the minimum segregation distance will be determined from an optimization procedure with respect to a "Targeted Risk Level". Different methods are considered: a brute force stepping method (when feasible), a standard Monte-Carlo method (only considered for the generation of reference results) and smart statistical methods for operatory solutions.

The standard Monte-Carlo method can assure high accuracy and ease of implementation. However, due to the fact that we are interested in evaluating a probability (of failure) having a very low value, it entails the analysis of a large number of random realizations.
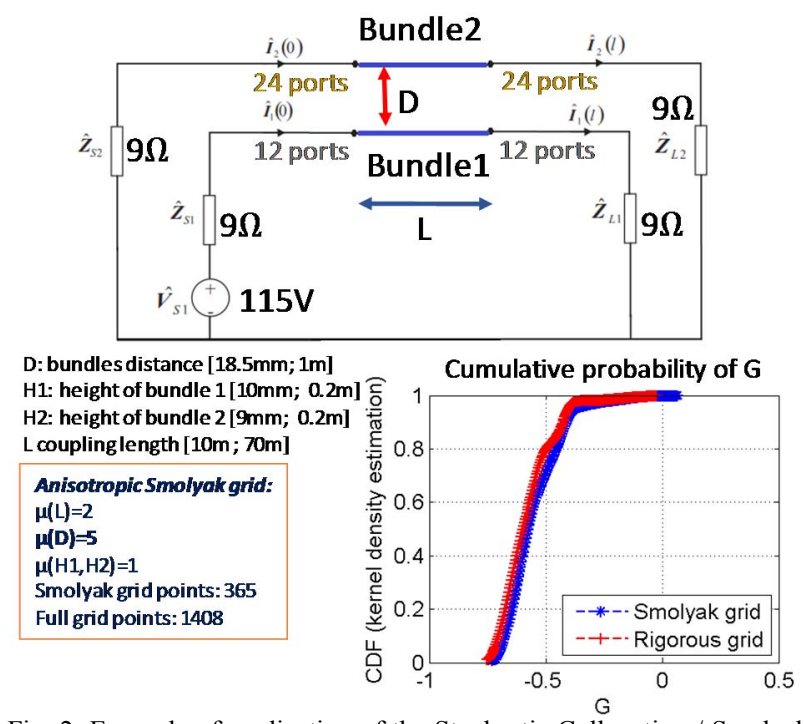

Fig. 2. Example of application of the Stochastic Collocation / Smolyak interpolation. Cumulative probability function of the $G$ quantity obtained analyzing 365 configurations of the transmission lines shown on the top, instead of the 1408 possible ones.

As efficient alternative, in order to analyze a minimal set of configurations, a stochastic collocation method based on Smolyak quadrature is considered ([3], [4]). The stochastic collocation method combines the Monte-Carlo approach with the polynomial approximation of stochastic quantities. It considers an interpolation over a pre-determined set of nodes in the stochastic space. The selection of interpolating functions and of the node selection strategy play a fundamental role in the accuracy of the method. Concerning the node selection, the conventional Smolyak method is isotropic, meaning that the same number of grid points and basis functions is used for all the variables in a symmetric way. Since our problem involves a large-scale system, it is useful to set separately the accuracy level of each variable in order to increase the quality of the approximation. For this, we adopted an anisotropic Smolyak grid, particularly effective in case of variables having different ranges or influences on the system.

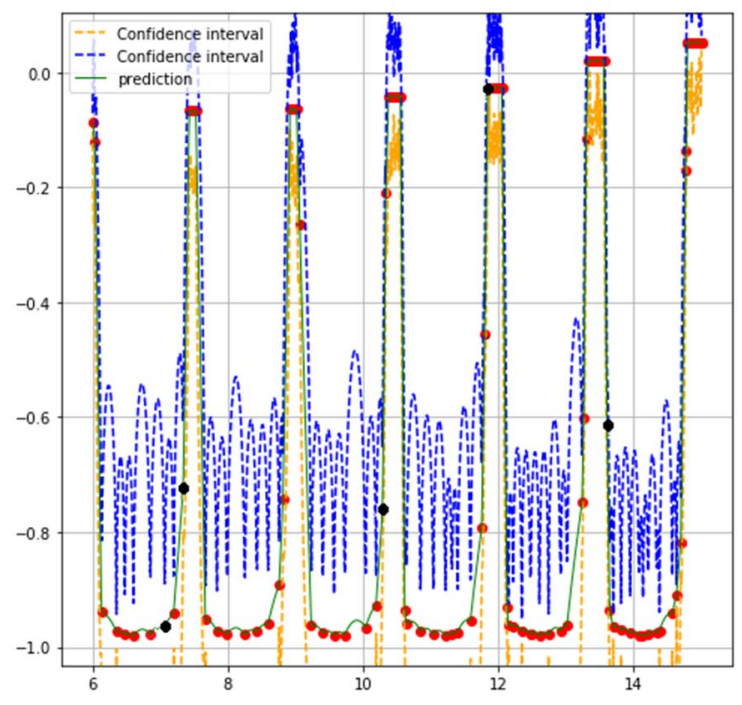

Fig. 3. Map of initial (black) and added sample points (red) for a complex problem involving one uncertain parameter to estimate the probability $\mathrm{P}(\mathrm{G}>0)$ with active learning reliability method. 
Just for example, Fig. 2 shows the result, in terms of cumulative probability of $G$ values, obtained analyzing the cable-network shown inset for 365 configurations instead of the 1408 full combinations.

Another alternative has been considered. It combines surrogate models and sampling strategies [5]. A surrogate model allows reduction of the number of configurations to be analyzed. Such an approach is particularly interesting in the case of rare event probability estimation, since the model has to be accurate not over the whole variation support of the parameters, but only in the failure region. In other words, the surrogate model does not have to be representative of the exact solution outside the regions of interest, as it does not influence the failure probability. For the analysis on the $G$ function, an active learning reliability method, a combination of Kriging and probability estimation methods is employed. Starting from a set of initial learning $G$ values, Kriging model is iteratively refined, by adding additional samples (meaning additional configurations analyzed by the MLTN solver), chosen by means of active learning [6]. Just for clarity purposes, Fig. 3 shows, for a problem having on one uncertain parameter, the initial and the added sample points in the refinement process to estimate the probability $P(G>0)$.

Both smart methods can give an accurate estimation of the behavior of the $G$ values. However, they can suffer in case of a wide range of variation of the coupling length involving large values and/or wide frequency sweep ranges leading to highly irregular $G$ functions. Especially, the presence of resonances can introduce a sharp variation on the $G$ values. This problem can be mitigated by introducing frequencyvariation losses in the transmission-line models (i.e. including $R(f)$ and $G(f)$ values in the per-unit-length (p.u.l.) parameters. Subdividing the frequency and coupling length ranges also contributes to improve the regularity of the $G$ function. Dedicated results will be shown at the conference.

\section{TOOL OPERATIONS}

The optimization of the segregation distances between a set of bundles by means of the statistical methods described in the previous section requires different calculation modules.

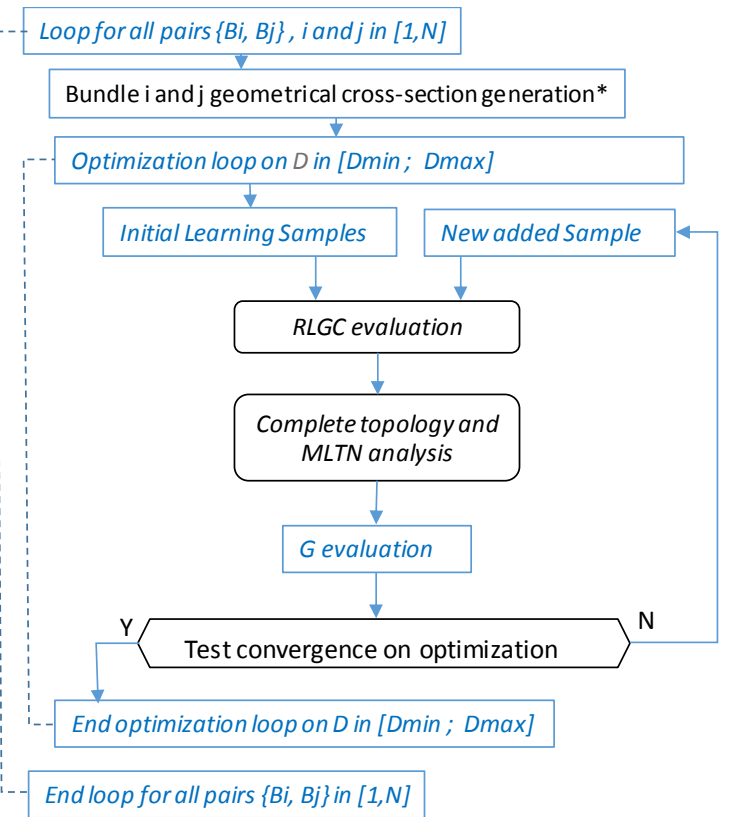

Fig. 1. Functional modules implementing the statistical analysis for the optimization of the segregation distance.

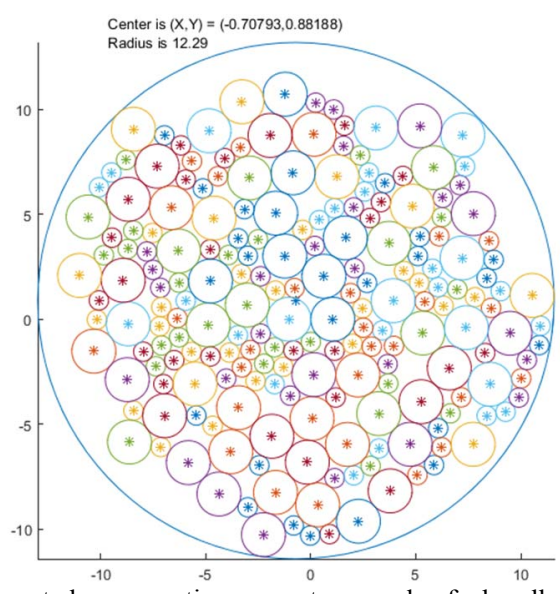

Fig. 5. Generated cross-section geometry sample of a bundle.

Fig. 4 sketches, from a functional point of view, the operations needed to perform a complete analysis. In the figure, black rectangular boxes refer to the needed modules, described in the following. It is worth to be noted that the same functional diagram applies also for Smolyak or standard Monte-Carlo analysis, providing that the initial learning samples constitute the Smolyak sampling grid and the total Monte-Carlo samples, respectively. In these cases, no added samples are needed.

We start from a complete description of the set of bundles and their generators, loads, and susceptibility levels with respect to frequency. Starting from a description of a bundle in terms of inner cables, an effective cross-section geometry of the bundles has to be generated. One or more random realizations of the cross-sections can be used, in order to account for the variability of the geometry of the bundles. We think it is important to maintain the same realizations over the entire analysis between all the bundles. The cross-section of two bundles will be combined in a third cross-section, depending from the installation parameters. This is needed to evaluate the p.u.l. RLGC parameters of the lines. The generation of a specified-cross section is basically a problem of circles packing. This problem has a large interest and several algorithms are described in literature, to obtain a good solution in a reasonable time. Different algorithms are available among the authors ([7], [8]) and are able to manage a large number of wires (Fig. 5).

A particular set of installation parameters defines how the two-bundles cross-sections have to be placed with respect to one another and with respect to the grounding structure. The MLTN analysis requires the evaluation of the p.u.l. RLGC parameters of the line for each different geometrical combination of the two bundle geometrical positions. This calculation is known to be significantly time consuming and, in past years, progress has been done to reduce the computational time by using GPGPU (General Purpose Graphic Processing Unit) implementation, with the drawback of a lower amount of available memory. The module used in this work (CLIG [9]) is able to use GPU if possible, or CPU, in an automatic way. Table I reports, as an example, the 


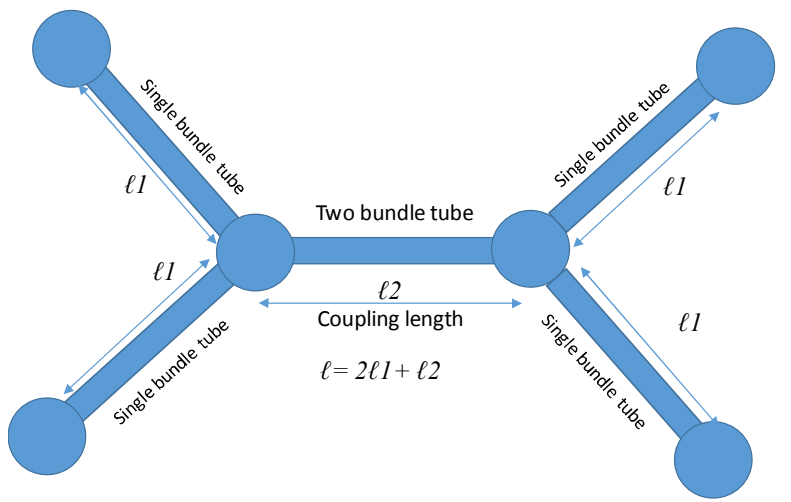

Fig. 6. Generic topology considered in the MLTN analysis for handling both parallel and crossing bundle configurations.

computational time required for different numbers of conductors in the combined cross-section.

TABLE 1

\begin{tabular}{|c|c|c|}
\hline \multirow{2}{*}{$\begin{array}{c}\text { Number of } \\
\text { conductors }\end{array}$} & \multicolumn{2}{|c|}{ CLIG RLGC computational time (min.) } \\
\cline { 2 - 3 } & GPU (ViennaCL) & $\boldsymbol{C P U}$ (Intel OpenCL) \\
\hline 5 & 0.003 & 0.022 \\
\hline 10 & 0.005 & 0.034 \\
\hline 35 & 0.010 & 0.180 \\
\hline 70 & 0.031 & 1.140 \\
\hline 350 & 2.17 & $2.8 \mathrm{~h}$ \\
\hline
\end{tabular}

The MLTN problem consisting of the combined bundles is analyzed with the CRIPTE BLT solver [10]. To allow the MLTN analysis also in the case of the crossing configuration of Fig.1, the H-topology shown in Fig. 6 is adopted. In this case, the "equivalent" coupling length depends from the intersecting angle, and from the other installation parameters.

\section{RESUlts}

In this section, we report some results obtained on a test case involving two parallel bundles over a planar ground plane. More results, involving some cases of interest from an industrial point of view, will be reported at the conference.

In any case, it is important to consider that the use of efficient techniques in the implemented statistical methods exploit interpolation (in our case, by using the Smolyak interpolation or a Kriging surrogate model). A particular

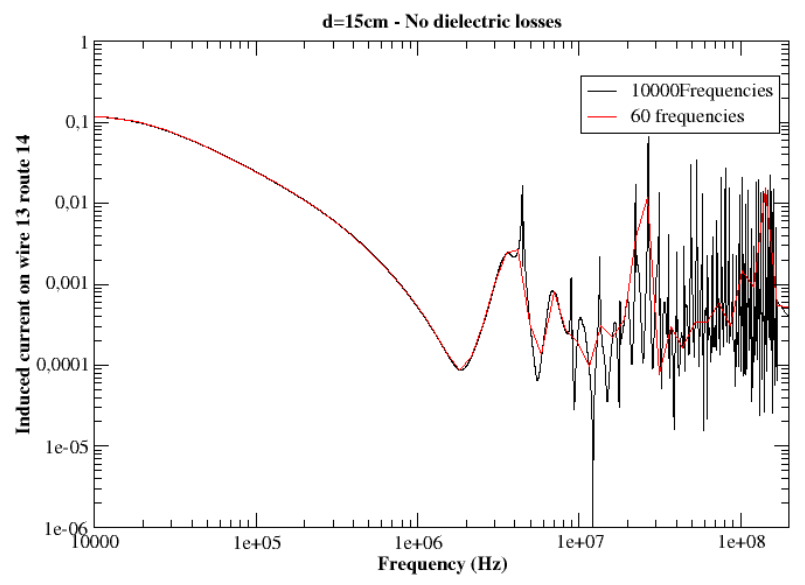

Fig. 7. Current induced on a wire of the victim bundle in case of a separation distance $D=15 \mathrm{~cm}$ between the two bundles of Fig. 2 .

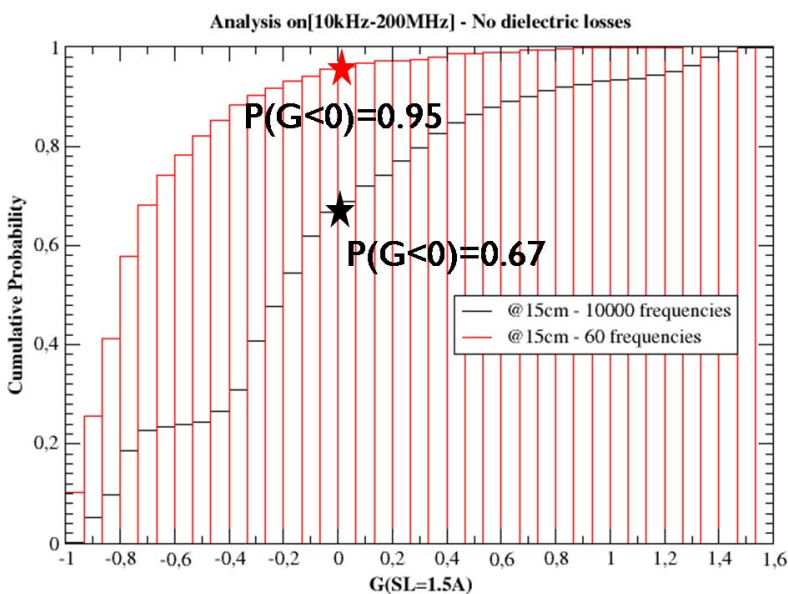

Fig. 8. Cumulative probability of the function $G$ for the case of Fig. 2 and a susceptibility level equal to $1.5 \mathrm{~A}$.

attention must be paid to the choice of the parameters of the methods, because the observed function ( $G$ in our case) can exhibit strong variations with variation range of the installation-parameters and/or in the frequency sweep. In this case, it can be very useful to split large ranges into smaller ones.

In addition, the inclusion of the real frequency dependent losses of the lines (ohmic, dielectric) into the RLGC parameters can be useful: their introduction have the effect of reducing the sharp behavior of the observed quantities around resonance regions.

In the following, we consider the configuration shown at the top of Fig. 2, calculated on a wide frequency range: from $10 \mathrm{kHz}$ to $200 \mathrm{MHz}$. The voltage generator is supposed constant over the whole frequency sweep.

Fig. 7 shows the current induced on a wire in the victim bundle when the separation of the two bundles is equal to $D$ $=15 \mathrm{~cm}$. If the number of sampling frequencies is too low, we miss the behavior of the cross-talk. Consequently, by using a standard Monte-Carlo method, we can miss a significant amount of resonances, resulting in an underestimation of the probability of a susceptible configuration, as shown in Fig. 8.

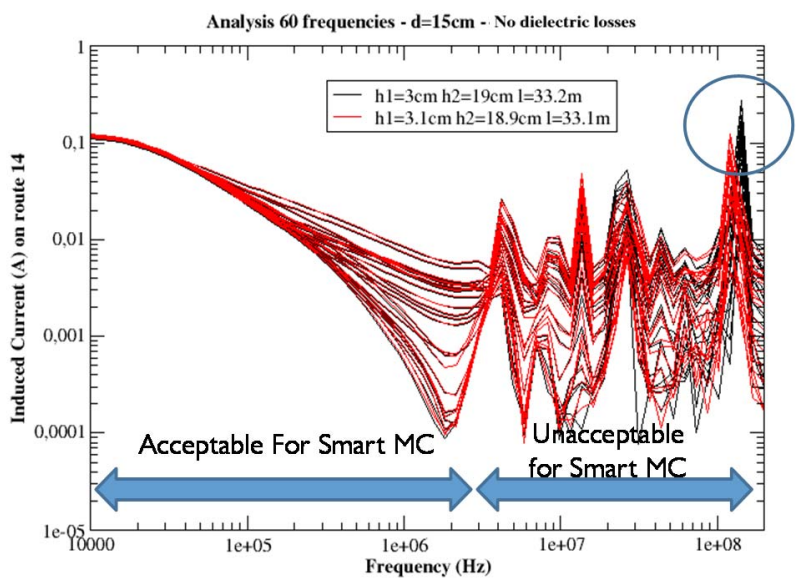

Fig. 9. Current induced on a wire of the victim bundle for a separation distance $D=15 \mathrm{~cm}$ between the two bundles of Fig. 2, for two very close configurations (the installation parameters are reported in the inset). 
Narrow Band Analysis @ Low Frequency (2MHz) $h_{A}=h_{B} \in[1.5 \mathrm{~cm}-20 \mathrm{~cm}] \mathrm{I} \in[6 \mathrm{~m}-15 \mathrm{~m}]$

\begin{tabular}{|c|c|c|}
\hline$d$ & $\begin{array}{c}\text { Kriging } \\
30+97 \text { points }\end{array}$ & Standard MC \\
\hline $5 \mathrm{~cm}$ & 1 & 1 \\
\hline $10 \mathrm{~cm}$ & 0.41 & 0.38 \\
\hline $13 \mathrm{~cm}$ & 0.14 & 0.13 \\
\hline $15 \mathrm{~cm}$ & 0.05 & 0.05 \\
\hline $20 \mathrm{~cm}$ & 0 & 0 \\
\hline $25 \mathrm{~cm}$ & 0 & 0 \\
\hline
\end{tabular}

Narrow Band Analysis @ High Frequency (100MHz) $h_{A}=h_{B} \in[1.5 \mathrm{~cm}-20 \mathrm{~cm}] \mathrm{I}=10 \mathrm{~m}$

\begin{tabular}{|c|c|c|}
\hline$d$ & $\begin{array}{c}\text { Kriging } \\
20+77 \text { points }\end{array}$ & Standard MC \\
\hline $5 \mathrm{~cm}$ & 1 & 1 \\
\hline $10 \mathrm{~cm}$ & 0.79 & 0.76 \\
\hline $13 \mathrm{~cm}$ & 0.41 & 0.41 \\
\hline $15 \mathrm{~cm}$ & 0.11 & 0.05 \\
\hline $20 \mathrm{~cm}$ & 0 & 0 \\
\hline $25 \mathrm{~cm}$ & 0 & 0 \\
\hline
\end{tabular}

Narrow Band Analysis @ High Frequency (100MHz)

\begin{tabular}{|c|c|c|}
\hline \multicolumn{2}{|c|}{$h_{A}=h_{B} \in[1.5 \mathrm{~cm}-20 \mathrm{~cm}]$} & $\mathrm{I} \in[6 \mathrm{~m}-15 \mathrm{~m}]$ \\
\hline $\mathrm{d}$ & Kriging & Standard MC \\
\hline $5 \mathrm{~cm}$ & $\mathrm{X}$ & 0.21 \\
\hline $10 \mathrm{~cm}$ & $\mathrm{X}$ & 0.14 \\
\hline $13 \mathrm{~cm}$ & $\mathrm{X}$ & 0.08 \\
\hline $15 \mathrm{~cm}$ & $\mathrm{X}$ & 0.05 \\
\hline $20 \mathrm{~cm}$ & $\mathrm{X}$ & 0 \\
\hline $25 \mathrm{~cm}$ & $\mathrm{X}$ & 0 \\
\hline
\end{tabular}

Fig. 10. Probability of failure $P(G>0)$ for different analysis of the network shown in Fig. 1. An X indicates a failed convergence in building of a surrogate model. $h_{A}$, and $h_{B}$ indicate the distance from the ground plane of the first and the second bundle, respectively. Brute MC refers to the standard Monte-Carlo approach.

The under-sampling of the resonance frequency region can cause also a very sharp variation of the $G$ function, as shown in Fig. 9. This usually results in a poor accuracy of the surrogated model employed with the Kriging approach. This happens also in case of the Smolyak approach, as reported in [4]. Pertinent inclusion of losses decreases of the quality factor of resonances and increases the accuracy of the smart methods.

Finding of the correct parameters to be used in the analysis to obtain good accuracy in the probability estimation represents the challenge of the current work.

In the following, we report some examples to show the effectiveness of the use of a surrogate model to perform the

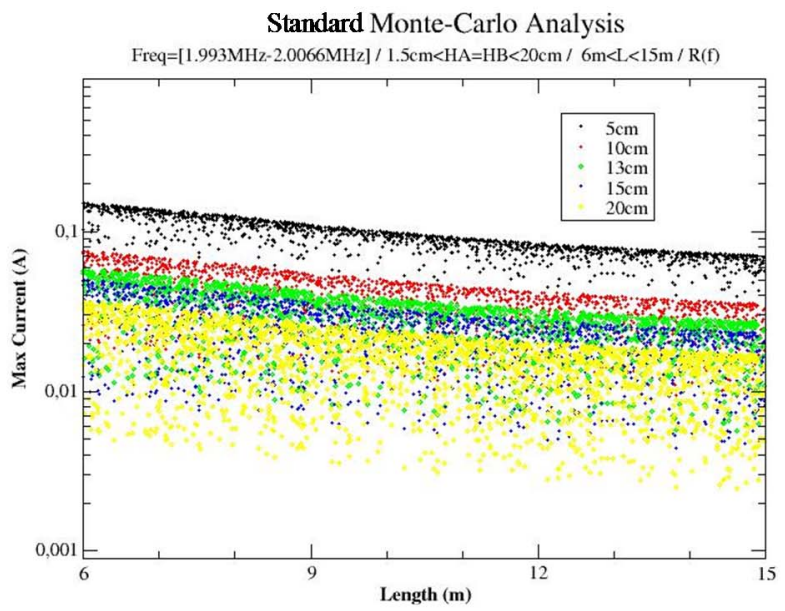

Standard Monte-Carlo Analysis

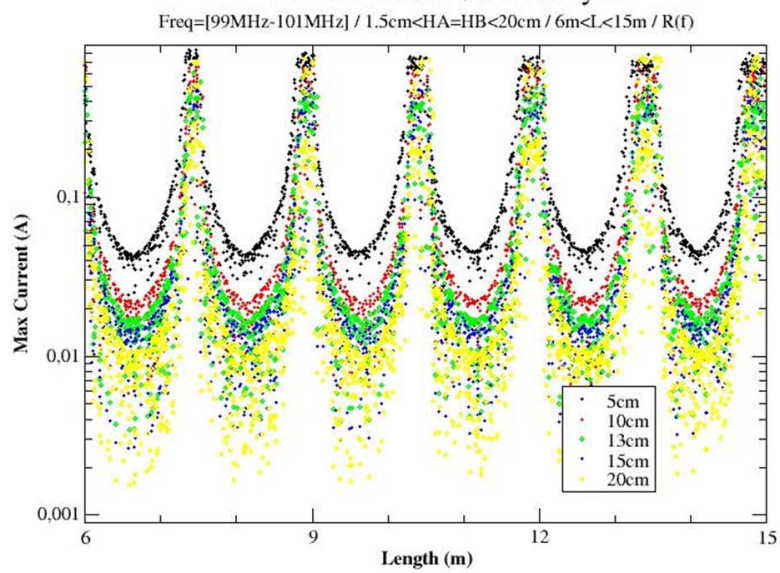

Fig. 11. Maximum induced current on victim cables on the configurations of the network shown in Fig.1, defined by the Monte Carlo samples. The graph at the bottom corresponds to the third case in Fig. 10, for which the building of a surrogate model does not reach convergence.

statistical analysis. We consider the network shown in Fig. 2 for different ranges of installation parameters and a relatively small frequency bands around a center frequency of $2 \mathrm{MHz}$ (low frequency) and $100 \mathrm{MHz}$ (high frequency).

Fig. 10 shows a comparison in terms of failure probability (meaning the probability to exceed the susceptibility threshold on at least one of the cables in the victim bundle) estimated by using a standard Monte-Carlo approach, and the smart approach combining surrogate model and sampling strategies described in Section III.

The first table of Fig. 10 concerns the comparison for the low frequency band and the whole range of installation parameters (height of bundles respect to the ground plane, and length of bundles). The building of a surrogate model requires 127 MLTN analysis, respect to the 10000 samples used in the standard Monte Carlo analysis, and the agreement is very good.

The second table of Fig. 10 concerns the comparison for the high frequency band and a fixed length of the two parallel bundles (while their height with respect to the ground plane varies on the whole range of values). The building of a surrogate model requires 97 MLTN analysis and, again in this case, the agreement is quite good. 
The third table of Fig. 10 concerns the comparison for the high frequency band and the whole range of installation parameters. In such a case and at this stage of our developments, the building of a surrogate model fails, meaning that a convergence criterion is not reached after a maximum number of samples (equals to 200 in these analyses). When we consider the whole range of variation of the length of the bundles, the function $G$ exhibits several maxima and minima; we are not able to build a surrogate model having a sufficient accuracy in a limited number of MLTN analysis. This is highlighted by Fig. 11, showing the maximum current among the cables of the victim bundle for the different set of installation parameters constituting the Monte Carlo sampling used in the estimation (each point represent a MLTN analysis performed for a standard Monte Carlo approach).

Due to these considerations, we modified the Tool in such a way that the statistical approach can switch to a standard Monte-Carlo algorithm if the building of an accurate surrogate model fails after a certain number of MLTN analysis. Moreover, we included also the possibility to perform independent statistical analysis on sub-intervals of the specified frequency sweep in order to mitigate the number of minima and maxima of the $\mathrm{G}$ function.

\section{ACKNOWLEDGMENT}

This paper is supported by European Union's Horizon 2020 research and innovation program, CFP07 GA 821128, project ANALYST (EM compatibility ANALYsis \& Statistical Techniques in aeronautics).

\section{REFERENCES}

[1] https://www.researchgate.net/project/ANALYST-EM-compatibilityANALYsis-Statistical-Techniques-in-aeronautics

[2] C. E. Baum, T. K. Liù, F. M. Tesche, "On the Analysis of General Multiconductor Transmission-Line Networks, Interaction Notes", Note 350, November 1978. Available at http://eceresearch.unm.edu/summa/notes/

[3] K. L. Judd, L. Maliar, S. Maliar and R. Valero, "Smolyak method for solving dynamic economic models: Lagrange interpolation, anisotropic grid and adaptive domain," Journal of Economic Dynamics \& Control, vol. 44, pp. 92-123, 2014

[4] M. D. Astorino, G. Antonini, et al., "Statistical Characterization of Segregation Distance Among Cable Bundles Aboard of Aircraft," to be published in 2020 International Symposium on Electromagnetic Compatibility - EMC EUROPE, Rome, 2020.

[5] Echard, B.: Kriging-based reliability assessment of structures submitted to fatigue. Ph.D. thesis, Université Blaise Pascal (2012).

[6] Echard, B., Gayton, N., Lemaire, M.: AK-MCS : An active learning reliability method combining Kriging and Monte-Carlo Simulation. Structural Safety 33, 145-154 (2011).

[7] H. Wang, W. Huang, Q. Zhang, D. Xu, "An improved algorithm for the packing of unequal circles within a larger containing circle," European Journal of Operational Research, 141(2), 440-453, 2002.

[8] W. Wang, H. Wang, G. Dai, and H. Wang, "Visualization of large hierarchical data by circle packing," in Proceedings of the SIGCHI Conference on Human Factors in Computing Systems (CHI '06). Association for Computing Machinery, New York, NY, USA, 517520, 2006.

[9] M. Ridel, J. P. Parmantier, C. Girard, C. Giraudon, "Methodology for modelling EM coupling on EWIS," 2012. Available at https://www.researchgate.net.

[10] J. P. Parmantier, S. Bertuol, and I. Junqua, "CRIPTE : Code de réseaux de lignes de transmission multiconducteur - User's guide - Version 5.1" ONERA/DEMR/T-N119/10 - CRIPTE 5.12010 